\title{
BOL AND EOL CHARACTERIZATION OF AZUR 3G LILT SOLAR CELLS FOR ESA JUICE MISSION
}

\author{
Victor Khorenko ${ }^{(1)}$, Carsten Baur ${ }^{(2)}$, Gerald Siefer ${ }^{(3)}$, Michael Schachtner ${ }^{(3)}$, Seonyong Park ${ }^{(4)}$, Bruno Boizot ${ }^{(4)}$, \\ Jacques C. Bourgoin ${ }^{(4)}$, Mariacristina Casale ${ }^{(5)}$, Roberta Campesato ${ }^{(5)}$ \\ (1) AZUR SPACE Solar Power GmbH, Theresienstr.2, 74072 Heilbronn, Germany, \\ Email:victor.khorenko@azurspace.com \\ (2) European Space Agency, Keplerlaan 1,2201AZ Noordwijk, The Netherland,Email: carsten.baur@esa.int \\ (3) Fraunhofer Institute for Solar Energy Systems, Freiburg, 79110, Germany,Email: gerald.siefer@ise.fraunhofer.de \\ (4) Laboratoire des Solides Irradiés, CNRS-UMR 7642, CEA-DRF-IRAMIS, Ecole Polytechnique, \\ Université Paris-Saclay, 91128 Palaiseau Cedex, France, Email:seonyong.park@polytechnique.edu \\ (5) CESI S.p.A., Milan, 20134, Italy, Email: mariacristina.casale@cesi.it
}

\begin{abstract}
In the present paper, we describe the results of electrical characterization of AZUR SPACE triple-junction solar cells at a sun light intensity of $3.7 \%$ AM0 and temperatures down to $-150^{\circ} \mathrm{C}$. At these conditions, which are relevant for the anticipated ESA JUICE mission, the cell efficiency reaches $33.5 \%$ at BOL. Special attention has been paid to the establishing of an in-situ characterization procedure for defining EOL cell characteristics after electron and proton irradiation at low temperature low intensity condition. It was shown that solar cells irradiated at low temperature exhibit a strong recovery effect within short time after stopping the irradiation whereas the absolute value of the recovery depends on the irradiation fluence and particle type. Further on, it was demonstrated that the degradation of the maximum power, Pmp, is much stronger than the degradation of Isc and Voc values. Experimentally defined remaining factors for electron and proton irradiation and the quantification of the observed recovery effects allow a realistic prediction of the solar cell performance at JUICE mission conditions and are essential for the planned solar cell qualification activities.
\end{abstract}

\section{INTRODUCTION}

The feasibility of solar cells for powering a spacecraft at a distance of up to five astronomic units from the sun has been successfully demonstrated with Si cells for the Rosetta mission and with triple-junction GaAs-based cells for the Juno mission. Notwithstanding, such deep space missions that combine low solar intensities with very low temperatures, which are often referred to as low intensity low temperature (LILT), likely pose the ultimate limit for photovoltaics technology in space.

The anticipated ESA JUpiter ICy moons Explorer (JUICE) mission will continuously operate at a light intensity and a temperature of $50 \mathrm{~W} / \mathrm{m}^{2}$ and $-130^{\circ} \mathrm{C}$, respectively. It has been shown that at LILT conditions solar cells become very sensitive to electrical shunts and may suffer from the so-called "flat spot" (or "broken knee") effect that may unpredictably appear and cause a drop of fill factor at temperatures below $-80^{\circ} \mathrm{C}$. Despite the increase of the cell voltage at low temperatures, both effects (shunts and flat spot) significantly lower the power output of the cell that is reduced due to low light intensity as well.

Shunts are caused by imperfections or defects in $p-n$ junctions within the solar cell structure or on the cell mesa edges. They are observable in dark IV measurements at room temperature and can be in principle significantly diminished by improving the overall manufacturing process. The appearance of the flat spot effect has been attributed to the formation of local Metal-Semiconductor-Like (MSL) junctions at the interface between the front side metallization and top sub-cell [1]. It was assumed that the formation of a MSL-junction occurs preferentially on local microscopic defects where crystal quality of the semiconductor material is disturbed. A general improvement of the cell epitaxy and manufacturing process and, as an additional measure, a suitable choice of the front grid metallization, have allowed for avoiding the flat spot effect completely [2, 3].

Another challenge consists in a reliable characterization of LILT solar cells, especially at End-of-Life (EOL) conditions. In contrast to the cells powering spacecraft in Earth orbit, the particle irradiation of LILT cells takes place at continuously lower temperatures. Thus, the determination of the EOL performance shall be preferable done at low temperatures in order to avoid any temperature annealing. This annealing will improve cell characteristics and lead to an overestimation of the EOL performance, which would be critical for designing of the spacecraft power sub-system.

In this paper, we present the latest results of the beginning-of-life (BOL) and end-of-life (EOL) electrical characterization of triple-junction AZUR solar cells and solar cell assemblies (SCAs) under LILT 
conditions. We paid special attention to the investigation of post-irradiation annealing und the quantification of annealing factors as a function of the irradiation fluence.

\section{MANUFACTURING AND SELECTION OF LILT SOLAR CELLS}

The solar cells were manufactured using AZUR 3G28 lattice matched triple junction solar cell structure based on the $\mathrm{InGaP} / \mathrm{InGaAs} / \mathrm{Ge}$ material combination. For $\mathrm{BOL}$ and EOL characterization after particle irradiation at room temperature, $4 \times 8 \mathrm{~cm}^{2}$ solar cells and solar cell assemblies (SCAs) were used, whereas the evaluation of the in-situ EOL performance and annealing effect study were done on $2 \times 2 \mathrm{~cm}^{2}$ bare cells fabricated using the same manufacturing processes. For all device designs, the cell thickness was chosen to be $80 \mu \mathrm{m}$.

Besides AZUR's standard quality inspection, all cells were subjected to additional acceptance tests which included room temperature electrical characterization at low light intensity and in the dark. The objective of these tests was to exclude solar cells with shunts from the later characterization; thus, the cells with fill factor $\mathrm{FF}<0.82$ and $\mathrm{I}_{\mathrm{fw}}>1 \mathrm{e}-2 \mathrm{~mA}$ at $\mathrm{V}_{\mathrm{fw}}=1.3 \mathrm{~V}$ (in the dark IV characteristic) were sorted out.

\section{TEST FACILITIES}

The pre-selection and room temperature characterization of solar cells and SCAs was performed by AZUR SPACE. BOL and EOL measurements of $4 \times 8 \mathrm{~cm}^{2}$ cells after room temperature irradiation were done at CESI, whereas BOL and EOL characterization of $2 \times 2 \mathrm{~cm}^{2}$ cells including EQE measurements at room and low temperatures was carried out by Fraunhofer ISE.

For the investigation of EOL performance at LILT conditions, the experimental setup of the Laboratoire des Solides Irradiés (LSI) of École Polytechnique in Palaiseau was used (Fig.1). The samples were placed inside a cryostat that was mounted onto a beam line of an irradiation facility. After finishing the irradiation, the cell holder is rotated towards a quartz window for illuminating the cell und recording its IV characteristic. To maintain the correct temperature during particle irradiation and subsequent measurements, the setup was preliminarily calibrated by comparing temperature records from the thermocouple built in the cell holder and a thermocouple glued to the surface of a test cell by a heat conductive adhesive.

Particle irradiation was performed with electrons and protons using SIRIUS irradiation facility of LSI and ARAMIS irradiation facility of CSNSM, respectively.

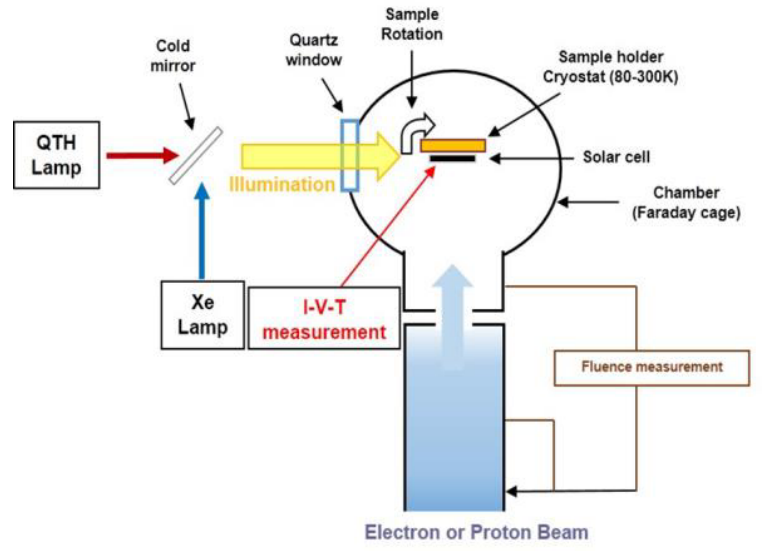

Figure 1. Experimental set-up for in-situ EOL characterisation at LILT conditions

\section{ELECTRICAL PERFORMANCE}

\subsection{Begin-of-Life Performance}

Electrical measurements performed at 3.7\%AM0 and temperatures down to $-150^{\circ} \mathrm{C}$ show an almost perfect shape of the IV characteristics with no signs of a flat spot effect as shown in Fig. 2.

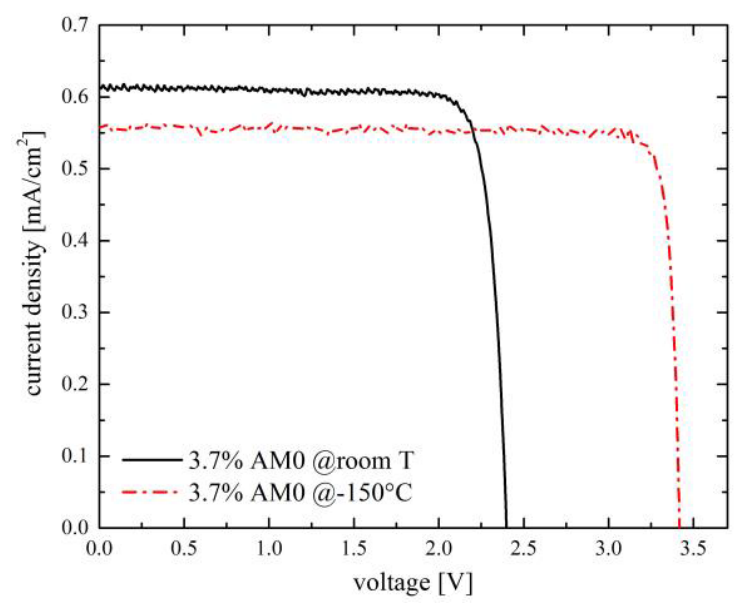

Figure 2. Low intensity (3.7\%AM0) IV characteristics of LILT solar cells at room temperature and $-150^{\circ} \mathrm{C}$

In terms of overall performance, low illumination intensity results in a BOL efficiency of approx. $25 \%$ at room temperature, which increases up to $33.5 \%$ at $-150^{\circ} \mathrm{C}$. Due to temperature effects, at LILT the cell voltage increases up to $3.41 \mathrm{~V}$. Solar cell characteristics averaged over 100 pcs of $4 \times 8 \mathrm{~cm}^{2}$ cells are given in Table 1. For comparison, standard AM0 data of the same cell group are presented, too. It is to note that the spread on BOL cell parameters at LILT was found to be the same as the one at standard AM0 conditions. 
Table 1. BOL performance of AZUR LILT solar cells at standard AMO and 3.7\% AMO room and low temperature conditions

\begin{tabular}{|ll|c|c|c|}
\hline \hline & & $\begin{array}{c}@ \text { AM0 } \\
28^{\circ} \mathrm{C}\end{array}$ & $@$ LIRT & @LILT \\
\hline \hline Jsc & {$\left[\mathrm{mA} / \mathrm{cm}^{2}\right]$} & 16.65 & 0.61 & 0.55 \\
\hline Voc & {$[\mathrm{V}]$} & 2.69 & 2.40 & 3.41 \\
\hline Pmp & {$[\mathrm{mW}]$} & 1178 & 38 & 51 \\
\hline FF & 0.87 & 0.86 & 0.9 \\
\hline Eta & {$[\%]$} & 28.5 & 25.1 & 33.5 \\
\hline \hline
\end{tabular}

As expected and suggested by the theory, the fill factor starts to increase linearly with lowering temperature. However, at temperatures below $-100^{\circ} \mathrm{C}$ this increase starts to saturate and at $-150^{\circ} \mathrm{C}$, typical fill factor values are in the range $0.89-0.91$ as shown in Fig. 3. This effect and its scatter is related to tunnelling effects that become the dominating contributors of the dark current at low temperatures [4].

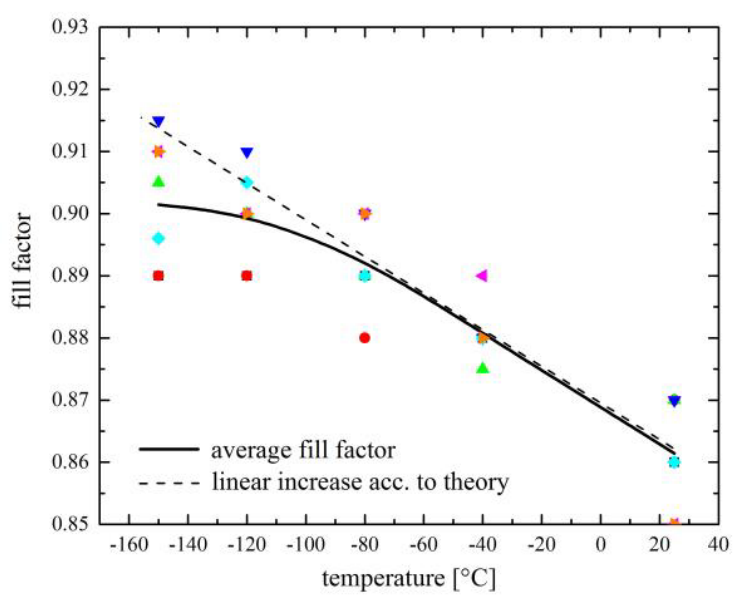

Figure 3. Temperature dependence of the fill factor at 3.7AMO.

\subsection{End-Of-Life (EOL) Performance}

As mentioned above, the main issue to be taken into account for the correct determination of the EOL performance is an appropriate test procedure that would allow for identifying the influence of temperature effects on the cell characteristics (annealing). The applied test procedure consisted in the irradiation of solar cells inside a cryostat at $-150^{\circ} \mathrm{C}$ and subsequent repetitive electrical measurements at $-150^{\circ} \mathrm{C}, 28^{\circ} \mathrm{C}$ and $150^{\circ} \mathrm{C}$ again. At each temperature, a series of IV curves was recorded to allow for observing time dependent changes of cell characteristics. Fig. 4 shows BOL and EOL IV characteristics of a triple-junction LILT solar cell irradiated with $3 \mathrm{e} 15 \mathrm{~cm}^{-2} 1 \mathrm{MeV}$ electrons at low temperature and measured in-situ at LILT conditions directly after stopping irradiation, after keeping the cell at these conditions for 30 minutes and after room temperature annealing. As can be seen, primary EOL cell characteristics (s. EOL1 curve in Fig 4) exhibits a noticeably recovery (EOL2 curve) within the first few minutes after irradiation, whereas keeping the cell at the same conditions longer than 10 minutes does not result in any further improvement (curves EOL3 and EOL4). However, after the cell annealing at higher temperatures, further recovery is observed (EOL-RA curve). Repetitive cell annealing at the same temperature does not change the cell characteristics anymore.

Thus, it can be concluded that a correct determination of the EOL solar cell performance at LILT conditions shall be based on in-situ measurements followed by the extraction of corresponding annealing coefficients. Knowing these coefficients would allow for avoiding very costly and time consuming low temperature irradiation testing during the solar cell qualification phase.

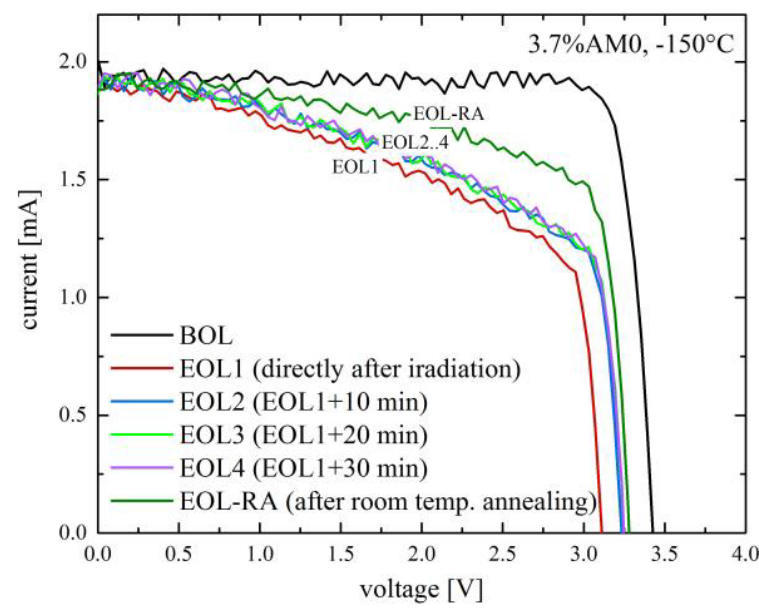

Figure 4. LILT BOL and EOL IV characteristics recorded directly after irradiation and after keeping the cell at LILT conditions for different time.

The second observation related to the behaviour of EOL solar cells at LILT conditions is that the most significant degradation occurs for Pmp compared to Isc and Voc values. A physical explanation of this "shunt"-like effect is given in [5].

The absolute value of the cell parameters recovery at LILT conditions depends on the irradiation fluence as illustrated in Fig. 5. After the highest applied $1 \mathrm{MeV}$ electron fluence of $3 \mathrm{e} 15 \mathrm{~cm}^{-2}$, the primary recovery (stabilization at low temperature) of Pmp consisted of approx. $6 \%$ and only approx. $2 \%$ after a fluence of $7.5 \mathrm{e} 14 \mathrm{~cm}^{-2}$. It is, however, to note that the recovery seems to be fast enough and, for the selected time scale, 
fluence independent: the stabilization was reached within 10 minutes in all measurements.

A similar dependence is observed for solar cells after room temperature annealing: up to $12 \%$ of improvement in Pmp after a fluence of $3 \mathrm{e} 15 \mathrm{~cm}^{-2}$ (compared to avg. Pmp value after stabilization) and approx. $2 \%$ after a fluence of $7.5 \mathrm{e} 15 \mathrm{~cm}^{-2}$.

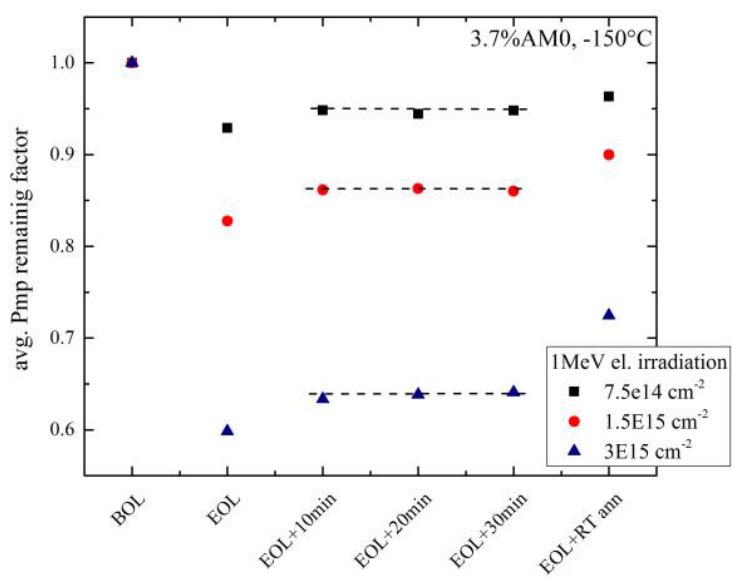

Figure 5. Recovery of LILT Pmp values after $1 \mathrm{MeV}$ electron irradiation with different fluences

For the practical application, the most importance have EOL LILT characteristics after stabilization as the particle irradiation in space has a flux of several orders of magnitude lower than the one used in tests and the generation of radiation defects in solar cells is in equilibrium with their possible recovery. Tab. 2 summarizes LILT remaining factors of solar cells measured after $1 \mathrm{MeV}$ electron irradiation and stabilization at low temperature. Similar data were also obtained for low temperature proton irradiation and are summarized in Tab. 3.

Table 2. Average LILT remaining factors measured at $3.7 \% \mathrm{AMO},-150^{\circ} \mathrm{C}$ after $1 \mathrm{MeV}$ electron irradiation and stabilization at LILT.

\begin{tabular}{|l|c|c|c|}
\hline \hline & $5 \mathrm{E} 14 \mathrm{~cm}^{-2}$ & $1 \mathrm{E} 15 \mathrm{~cm}^{-2}$ & $2 \mathrm{E} 15 \mathrm{~cm}^{-2}$ \\
\hline \hline RF Isc & 0.99 & 0.96 & 0.89 \\
\hline RF Voc & 0.95 & 0.93 & 0.91 \\
\hline RF Pmp & 0.89 & 0.76 & 0.66 \\
\hline RF Eta & 0.89 & 0.76 & 0.66 \\
\hline \hline
\end{tabular}

In order to verify the remaining factors obtained in the in-situ measurements, BOL and EOL characterization at LILT conditions was also performed at Fraunhofer ISE. As the EOL cells measured at ISE did receive unavoidable room temperature annealing during the transfer from the irradiation facility, their EOL data were corrected using annealing coefficients extracted from in-situ data.

Table 3. Average LILT remaining factors measured at $3.7 \% \mathrm{AMO},-150^{\circ} \mathrm{C}$ after $1 \mathrm{MeV}$ proton irradiation and stabilization at LILT.

\begin{tabular}{|l|c|c|c|}
\hline \hline & $1 \mathrm{e} 11 \mathrm{~cm}^{-2}$ & $2 \mathrm{e} 11 \mathrm{~cm}^{-2}$ & $4 \mathrm{e} 11 \mathrm{~cm}^{-2}$ \\
\hline \hline RF Isc & 0.97 & 0.95 & 0.86 \\
\hline RF Voc & 0.94 & 0.92 & 0.91 \\
\hline RF Pmp & 0.86 & 0.82 & 0.73 \\
\hline RF Eta & 0.86 & 0.85 & 0.73 \\
\hline \hline
\end{tabular}

One issue is not completely understood, yet. Whereas the distribution of LILT cell performance at BOL is relatively narrow and corresponds to this one at standard AM0 conditions, EOL cell parameters both, after stabilization and room temperature annealing, exhibit a much wider spread that magnitude correlates with the irradiation fluence (Fig. 6). Furthermore, the observed dependence of this spread on the kind of particles (electrons or protons) suggests a dedicated role of particle scattering mechanisms and distribution of irradiation defects in the cell material.

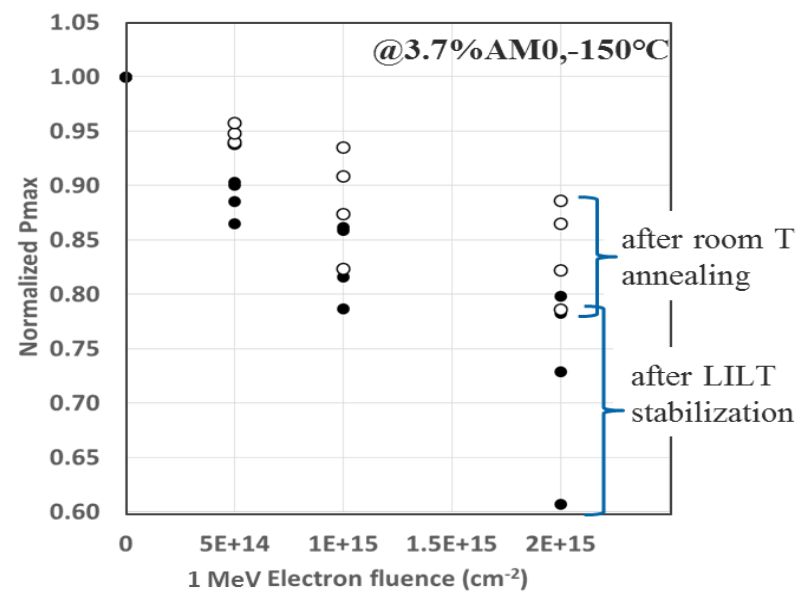

Figure 6. Spread of Pmp values at LILT after $1 \mathrm{MeV}$ electron irradiation with different fluences

\section{CONCLUSION}

In conclusion, we performed extensive BOL and EOL characterization of $3 \mathrm{G} 28$ triple-junction solar cells at LILT conditions relevant for a mission to planet Jupiter. At $3.7 \%$ AM0 and $-150^{\circ} \mathrm{C}$, AZUR LILT solar cells show a superior efficiency of $33.5 \%$. The need in 
correct determination of the EOL performance under these specific conditions led to the establishing of a sophisticated test procedure to avoid temperature related annealing effects. Using in-situ IV measurements, it was demonstrated that the degradation of Pmp of solar cells irradiated at low temperature is much stronger than the degradation of Isc and Voc values. It was also shown that solar cells irradiated at low temperatures exhibit a strong recovery effect within short time after the irradiation. The absolute value of the recovery depends on the irradiation fluence and particle type. For a fluence of $3 \mathrm{e} 15 \mathrm{~cm}^{-2} 1 \mathrm{MeV}$ electrons, the recovery of Pmp at low temperature consisted of $\sim 6 \%$ and the room temperature annealing - of $12 \%$. Experimentally defined remaining factors for electron and proton irradiation allow for a realistic prediction of the solar cell performance at JUICE mission conditions whereas the quantification of annealing effects is essential for the planned cell qualification activity.

\section{ACKNOWLEDGMENTS}

Present work was supported by the European Space Agency under contract no. 4000109645.

\section{REFERENCES}

1. V. G. Weizer and J. D. Broder, "On the cause of the flat-spot phenomenon observed in silicon solar cells at low temperatures and low intensities", Journal of Applied Physics 53(8), pp. 5926-5930 (1982)

2. V. Khorenko, G.F.X. Strobl, R. Hoheisel, F. Dimroth, R. Campesato, M. Casale, C. Baur, "Triple-junction solar cell design for low intensity low temperature space applications", Proc. of the 9th European Space Power Conference ESPC, ISBN 978-92-9092-257-5, France (2011)

3. V. Khorenko, C. Baur, G. Siefer , J.C. Bourgoin R. Campesato, M. Casale, "Performance analysis of AZUR 3G28 triple-junction solar cells optimized for operation in Jupiter environment". Proc. of the 10th European Space Power Conference ESPC, the Netherlands (2014)

4. R. Hoheisel, R. J. Walters, and A. W. Bett, "Low temperature effects in photovoltaic devices for deep space missions," in Proceedings of the 42nd IEEE Photovoltaic Specialist Conference, 2015, pp. 1-5.

5. S. Park, J. C. Bourgoin, O. Cavani, V. Khorenko, C. Baur, and B. Boizot, "Origin of the degradation of triple junction solar cells at low temperature," at this conference. 